\title{
On Absolute Summability for any Positive Order
}

\author{
By C. E. Wixn (Egyptian University). \\ (Received 24th May, 1932. Read 4th June, 1932.)
}

§1. Absolute summability according to Cesàro's method has been defined by Fekete ${ }^{1}$ for positive integral orders, as follows :-

Denoting the $r$ th partial sum of a series $\Sigma u_{n}$ by $S_{n}^{r}$ and its $r$ th mean, namely $S_{n}^{r} / A_{n}^{r}$, by $s_{n}^{r}$, we can regard $s_{n}^{r}$ as the sum of the series

$$
\sum_{\nu=0}^{n} u_{\nu}^{r} \equiv \sum_{\nu=0}^{n}\left(s_{v}^{r}-s_{\nu-1}^{r}\right) \quad\left(s_{-1}^{r}=0\right)
$$

Thus the convergence of $\Sigma u_{i n}^{r}$ is equivalent to summability $(C, r)$. If now this series is absolutely convergent, we say that $\Sigma u_{n}$ is absolutely summable $(C, r)$, or summable $|C, r|$.

The above definition can be adapted without change for nonintegral orders; it is the object of the present paper to extend to all positive orders the consistency and product results given by Fekete. As a preliminary investigation bearing on the question appears an analogue of Toeplitz's theorem for sequences of bounded variation, the sufficiency only, and not the necessity, being taken into consideration.

§2. If $s_{n}$ is a sequence of bounded variation, i.e. for which

$$
\Sigma u_{n}=\Sigma\left(s_{n}-s_{n-1}\right)
$$

is absolutely convergent, then

$$
t_{n}=\sum_{\nu=0}^{n} a_{n, \nu} s_{\nu} \quad\left(a_{n, \nu}=0, \text { if } \nu>n\right)
$$

is also of bounded variation, provided that the double sum

for all values of $N$.

$$
\sum_{\bullet \leqq \nu \leqq n \leqq N}\left|a_{n, \nu}-a_{n-1, \nu}\right|<K
$$

1 Math. ès Term. Ert. (1911), pp. 719.726.

$2 A_{n}^{r}$ stands for $\left(\begin{array}{c}n+r \\ n\end{array}\right)$. 
For we have

and

$$
\begin{aligned}
v_{n}=t_{n}-t_{n-1} & =\sum_{\nu=0}^{n}\left(a_{n, v}-a_{n-1, v}\right)\left(u_{0}+u_{1}+\ldots+u_{v}\right) \\
& =\sum_{i=0}^{n} u_{i} \sum_{\nu=i}^{n}\left(a_{n, v}-a_{n-1, v}\right)
\end{aligned}
$$

$$
\begin{aligned}
\sum_{n=0}^{N}\left|v_{n}\right| & \leqq \sum_{n=0}^{N} \sum_{i=0}^{n}\left|u_{i}, \sum_{v=i}^{n}\right| a_{n, v}-a_{n-1, \nu} \mid \\
& =\sum_{i=0}^{N}\left|u_{i}\right| \sum_{i \leqq \nu \leqq n \leqq N}\left|a_{n, v}-a_{n-1, \nu}\right| \\
& <K \sum_{i=0}^{N}\left|u_{i}\right| .
\end{aligned}
$$

A simple case presents itself, when

For then

$$
\sum_{\nu=i}^{n}\left(a_{n, v}-a_{n-1, \nu}\right) \geqq 0 \quad(0 \leqq i \leqq n) .
$$

$$
\begin{aligned}
\left|v_{n}\right| & \leqq \sum_{i=0}^{n}\left|u_{i}\right| \sum_{v=i}^{n}\left(a_{n, v}-a_{n-1, v}\right) \\
& =\sum_{v=0}^{n 2}\left(a_{n, v}-a_{n-1, v}\right) \sigma_{\nu},
\end{aligned}
$$

where $\sigma_{\nu}=\sum_{i=0}^{\nu}\left|u_{i}\right| . \quad$ It follows that

$$
\sum_{n=0}^{N}\left|v_{n}\right| \leqq \sum_{\nu=0}^{N} a_{N, \nu} \sigma_{\nu}
$$

Consequently, if the ordinary Toeplitz conditions hold, we can deduce, without assuming (1), that $\Sigma v_{n}$ converges absolutely.

$\S 3$. We next prove two lemmas.

LEMMA I.

$$
\frac{1}{A_{n}^{p+q}+1} \sum_{\nu=i}^{n-j} A_{\nu}^{p \prime} A_{n-\nu}^{q}
$$

does not increase as $n$ increases $(p>-1, q>-1, p+q>-1 ; i, j, \geqq 0)$. We have

$$
0<\alpha_{p}=\frac{A_{v}^{p} A_{n-1}^{q}}{A_{n}^{p+q+1}} \lesseqgtr \frac{A_{v}^{p} A_{n-v-1}^{q}}{A_{n-1}^{p+q+1}}=\beta_{v},
$$

according as

$$
\nu \supseteqq \frac{p+1}{p+q+1} n=\nu_{1}, \text { say. }
$$


Hence, for $0 \leqq \nu \leqq \nu_{1}$,

$$
a_{0}+a_{1}+\ldots+a_{v}<\beta_{0}+\beta_{1}+\ldots+\beta_{v} ;
$$

and for $\nu_{1}<\nu<n_{\ddagger}$ noting that

we get

$$
\sum_{v=0}^{n} a_{v}=\sum_{v=0}^{n} \beta_{v}=1
$$

$$
\begin{aligned}
a_{0}+\alpha_{1}+\ldots+\alpha_{\nu} & =1-\left(\alpha_{\nu+1}+\alpha_{\nu+2}+\ldots+\alpha_{n}\right) \\
& <1-\left(\beta_{\nu+1}+\beta_{\nu+2}+\ldots+\beta_{n-1}\right) \\
& =\beta_{0}+\beta_{1}+\ldots+\beta_{\nu} .
\end{aligned}
$$

Therefore

$$
\frac{1}{A_{n}^{p+1+1}} \sum_{v=0}^{i-1} A_{v}^{i j} A_{n-1}^{i}
$$

decreases, as $n$ increases $(i>0)$. And in the same way

$$
\frac{1}{A_{n}^{p+q+1}} \sum_{\nu=0}^{j-1} A_{v}^{q} A_{n-v}^{p}
$$

decreases, as $n$ increases $(j>0)$. Our result thus follows, since ${ }^{1}$

$$
\frac{1}{A_{n}^{p+q+1}} \sum_{\nu=i}^{n-j} A_{\nu}^{p} A_{n-\nu}^{q}=\frac{1}{A_{n}^{p+q+1}}\left\{\sum_{\nu=0}^{n}-\sum_{\nu=0}^{i-1}-\sum_{\nu=n \rightarrow-1}^{n}\right\} A_{v}^{p} A_{n-\nu}^{q} .
$$

Lemma II.

$$
A_{j}^{l} \sum_{\nu=0}^{\infty} \frac{A_{v}^{k-1}}{A_{j+\nu}^{k+l}}
$$

is finite for all $j \geqq 0, k \geqq 0, l>0$.

When $k=0$, the sum reduces to unity. Otherwise we have $\sum_{v=0}^{i} A_{v}^{k-1}=A_{j}^{k}$, and as $i$ becomes great,

so that

$$
A_{i}^{r} \cong \frac{i^{r}}{\Gamma(r+1)}(r>-1)
$$

$$
\begin{aligned}
\sum_{\nu=0}^{\infty} \frac{A_{\nu}^{k-1}}{A_{j+\nu}^{k+l}} & =\left(\sum_{\nu=0}^{j}+\sum_{\nu=j+1}^{\infty}\right) \frac{A_{\nu}^{k-1}}{A_{j+\nu}^{k+l}} \\
& >\frac{A_{j}^{k}}{A_{j}^{k+l}}+O \sum_{\nu=j+1}^{\infty}\left(\frac{\nu}{j+\nu}\right)^{k-1} \frac{1}{(j+\nu)^{l+1}}=O\left(\frac{1}{j^{l}}\right),
\end{aligned}
$$

1 If $i$ or $j$ is zero, we omit the corresponding sum. 
which yields the required result. We conclude with our two theorems.

§4. THEOREM I. If $\Sigma u_{n}$ is summable $|C, k|$, then it is also summable $|C, k+l|, \quad(k \geqq 0, \quad l>0)$.

We have

$$
\begin{aligned}
s_{n}^{k+l}=\frac{S_{n}^{k+l}}{A_{n}^{k+l}} & =\sum_{\nu=0}^{n} \frac{A_{n-\nu}^{l-1} A_{\nu}^{k}}{A_{n}^{k+l}} s_{\nu}^{k} \\
& =\sum_{\nu=0}^{n} a_{n_{\nu}} s_{\nu}^{k}, \text { say } ;
\end{aligned}
$$

and from Lemma $I$, with $j=0$, it appears that ${ }^{1}$

$$
\sum_{\nu=i}^{n}\left(a_{n, \nu}-a_{n-1, \nu}\right) \geqq 0 .
$$

Consequently, in view of our earlier remarks, the series $\Sigma u_{n}^{k+l}$ converges absolutely.

THeorem II. If $\Sigma u_{n}$ is summable $|C, k|$ and $\Sigma v_{n}$ is summable $|C, l|$, then the product series

$$
\Sigma w_{n} \equiv \Sigma\left(u_{0} v_{n}+u_{1} v_{n-1}+\ldots+u_{n} v_{0}\right)
$$

is summable $|C, k+l|, \quad(k \geqq 0, l \geqq 0)$.

When $k$ and $l$ are both zero, this reduces to the well-known theorem on the multiplication of two absolutely convergent series. We may suppose then that $l>0$. Denoting the partial sums of order $r$ for $\Sigma u_{n}$ and $\Sigma v_{n}$ by $S_{n}^{r}$ and $T_{n}^{r}$ respectively, we have, when $|x|<1$,

$$
\begin{aligned}
(1-x)^{-k-l-1} \sum_{n=0}^{\infty} u_{n} x^{n} \cdot \sum_{n=0}^{\infty} v_{n} x^{n} & =(1-x)^{-k} \sum_{n=0}^{\infty} u_{n} x^{n} \cdot(1-x)^{-l-1} \sum_{n=0}^{\infty} v_{n} x^{n} \\
& =\sum_{n=0}^{\infty} S_{n}^{k-1} x^{n} \cdot \sum_{n=0}^{\infty} T_{n}^{l} x^{n} .
\end{aligned}
$$

Hence the partial sum of order $k+l$ for the product series is

$$
\begin{aligned}
P_{n}^{k+l} & =\sum_{\nu=0}^{n}\left(S_{\nu}^{k}-S_{\nu-1}^{k}\right) T_{n-\nu}^{l} \\
& =\sum_{\nu=0}^{n}\left(A_{\nu}^{k} s_{\nu}^{k}-A_{\nu-1}^{k} s_{\nu-1}^{k}\right) A_{n-\nu}^{l} t_{n-\nu}^{l}
\end{aligned}
$$

${ }^{1} a_{n-1, n}$ vanishes, if we take $A_{-1}^{r}=0(r=0,-1,-2 \ldots)$. This follows, if we suppose $A_{n-1}^{r}=A_{n}^{r} \frac{n}{n+r}$ true for $n=0$. 
and

$$
\begin{aligned}
w_{n}^{k+l} & =\frac{P_{n}^{k+l}}{A_{\nu}^{k+l}}-\frac{P_{n-1}^{k+l}}{A_{n-1}^{k+l}} \\
& =\sum_{\nu=0}^{n}\left(A_{\nu}^{k} s_{\nu}^{k}-A_{\nu-1}^{k} s_{\nu-1}^{k}\right)\left(\frac{A_{n-\nu}^{l} l_{n-\nu}^{l}}{A_{n}^{k+l}}-\frac{A_{n-\nu-1}^{l} t_{n-\nu-1}^{l}}{A_{n-1}^{k+l}}\right) \\
& =\sum_{\nu=0}^{n}\left(A_{\nu}^{k-1} s_{\nu}^{k}+A_{\nu-1}^{k} u_{\nu}^{k}\right)\left\{\left(\frac{A_{n-\nu}^{l}}{A_{n}^{k+l}}-\frac{A_{n-\nu-1}^{l}}{A_{n-1}^{k+l}}\right) t_{n-\nu}^{l}+\frac{A_{n-\nu-1}^{l}}{A_{n-1}^{k+l}} v_{n-\nu}^{l}\right\} \\
& =\sum_{\nu=0}^{n} A_{\nu}^{k-1} s_{\nu}^{k}\left(\frac{A_{n-\nu}^{l}}{A_{n}^{k+l}}-\frac{A_{n-\nu-1}^{l}}{A_{n-1}^{k+l}}\right) t_{n-\nu}^{l}+\sum_{\nu=0}^{n-1} A_{\nu}^{k-1} s_{\nu}^{k} \frac{A_{n-\nu-1}^{l}}{A_{n-1}^{k+l}} v_{n-\nu}^{l} \\
& +\sum_{\nu=1}^{n} A_{\nu-1}^{k} u_{\nu}^{k}\left(\frac{A_{n-\nu}^{l}}{A_{n}^{k+l}}-\frac{A_{n-\nu-1}^{l}}{A_{n-1}^{k+l}}\right) t_{n-\nu}^{l}+\sum_{\nu=0}^{n-1} A_{\nu-1}^{k} u_{\nu}^{k} \frac{A_{n-\nu-1}^{l}}{A_{n-1}^{k+l}} v_{n-\nu}^{l} \\
& =\sum_{1}+\Sigma_{2}+\sum_{3}+\Sigma_{4}, \quad \text { say. }
\end{aligned}
$$

Now

$$
\begin{aligned}
\Sigma_{1} & =\sum_{\nu=0}^{n} A_{\nu}^{k-1} \sum_{i=0}^{\nu} u_{i}^{l} \sum_{j=0}^{n-\nu} v_{j}^{l}\left(\frac{A_{n-\nu}^{l}}{A_{n}^{k+l}}-\frac{A_{n-\nu-1}^{l}}{A_{n-1}^{k+l}}\right) \\
& =\sum_{0 \leqq i+j \leqq n} u_{i}^{k} v_{j}^{l}\left\{\sum_{v=i}^{n-j} \frac{A_{v}^{k-1} A_{n-\nu}^{l}}{A_{n}^{k+l}}-\sum_{\nu=i}^{n-j-1} \frac{A_{\nu}^{k-1} A_{n-\nu-1}^{l}}{A_{n-1}^{k+l}}\right\} .
\end{aligned}
$$

But the expression in brackets is $\geqq 0$ by Lemma $I$. Hence

$$
\begin{aligned}
\left|\Sigma_{1}\right| \leqq & \sum_{0 \leqq i+j \leqq n}\left|u_{i}^{k}\right|\left|v_{j}^{l}\right|\left\{\sum_{\nu=i}^{n-j} \frac{A_{\nu}^{k-1} A_{n-\nu}^{l}}{A_{n}^{k+l}}-\sum_{\nu=i}^{n-j-1} \frac{A_{\nu}^{k-1} A_{n-\nu-1}^{l}}{A_{n-1}^{k+l}}\right\}, \\
\sum_{n=1}^{N}\left|\Sigma_{1}\right| & \leqq \sum_{0 \leqq i+j \leqq N}\left|u_{i}^{k}\right|\left|v_{j}^{l}\right| \sum_{\nu=i}^{N-j} \frac{A_{\nu}^{k-1} A_{N-\nu}^{l}}{A_{N}^{k+l}} \\
& \leqq \sum_{0 \leqq i+j \leqq N}\left|u_{i}^{k}\right|\left|v_{j}^{l}\right| \leqq \sum_{i=0}^{\infty}\left|u_{i}^{k}\right| \sum_{j=0}^{\infty}\left|v_{j}^{l}\right|=O(1) .
\end{aligned}
$$

Next, using Lemma II, we get, when $\bar{s}$ is the upper bound of $\left|s_{\nu}\right|$,

$$
\begin{aligned}
\sum_{n=1}^{N}\left|\Sigma_{2}\right| & \leqq \bar{s} \sum_{n=1}^{N} \frac{1}{A_{n}^{k+l}} \sum_{\nu=0}^{n-1} A_{\nu}^{k-1} A_{n-\nu-1}^{l}\left|v_{n-\nu}^{l}\right| \\
& =\bar{s} \sum_{j=1}^{N}\left|v_{j}^{l}\right| O(1)=O(1) .
\end{aligned}
$$

In $\Sigma_{3}$ we notice that, for $k>0$,

$$
\left|\frac{A_{n-\nu}^{l}}{A_{n}^{k+l}}-\frac{A_{n-\nu-1}^{l}}{A_{n-1}^{k+l}}\right|=\left|\frac{A_{n-\nu}^{l-1}}{A_{n}^{k+l}}-\frac{k+l}{n} \frac{A_{n-\nu-1}^{l}}{A_{n}^{k+l}}\right|<K \frac{A_{n-\nu}^{l-1}}{A_{n}^{k+l}}
$$

1 It should be observed that $\Sigma_{1}$ and $\Sigma_{2}$ do not occur when $k=0$. 
and, for $k=0$, the expression is equal to

$$
\frac{\nu A_{n-v}^{l-1}}{n A_{n}^{l}}<K^{\prime} \frac{A_{\nu-1}^{1} A_{n-\nu}^{l-1}}{A_{n}^{i+1}}
$$

$\Sigma_{3}$ can therefore be treated like $\Sigma_{2}$, so that $\left.\sum_{n=1}^{N} \mid \Sigma_{3}\right\}=O(1)$.

Finally

$$
\begin{aligned}
& \left|\Sigma_{4}\right| \leqq \sum_{\nu=0}^{n-1}\left|u_{\nu}^{k}\right|\left|v_{n-\nu}^{l}\right| \\
& \sum_{n=1}^{N}\left|\Sigma_{4}\right| \leqq \sum_{\nu=0}^{\infty}\left|u_{\nu}^{k}\right| \sum_{\nu=0}^{\infty}\left|v_{\nu}^{l}\right|=O(1)
\end{aligned}
$$

Combining results we see that $\sum_{n=1}^{N}\left|w_{n}^{k+l}\right|$ is finite for all $N$, i.e. that the product series is summable $|C, k+l|$. 